\title{
Evidence of large magneto-dielectric effect coupled to a metamagnetic transition in $\mathrm{Yb}_{2} \mathrm{CoMnO}_{6}$
}

\author{
J. Blasco, ${ }^{1, a)}$ J. L. García-Muñoz, ${ }^{2}$ J. García, ${ }^{1}$ J. Stankiewicz, ${ }^{1}$ G. Subías, ${ }^{1}$ C. Ritter, ${ }^{3}$ \\ and J. A. Rodríguez-Velamazán ${ }^{1,3}$ \\ ${ }^{1}$ Instituto de Ciencia de Materiales de Aragón, CSIC-Universidad de Zaragoza, 50009 Zaragoza, Spain \\ ${ }^{2}$ Institut de Ciència de Materials de Barcelona, ICMAB-CSIC, Campus Univ. de Bellaterra, \\ E-08193 Bellaterra, Spain \\ ${ }^{3}$ Institute Laue Langevin, BP 156, 38042 Grenoble Cedex 9, France
}

(Received 10 April 2015; accepted 25 June 2015; published online 6 July 2015)

\begin{abstract}
The double perovskite $\mathrm{Yb}_{2} \mathrm{CoMnO}_{6}$ has been synthesized with an almost perfect checkerboard arrangement of $\mathrm{Co}^{2+}$ and $\mathrm{Mn}^{4+}$ cations in the B-sublattice of the perovskite cell. It presents an anomaly in the electric capacitance and a strong magneto-dielectric effect at about $40 \mathrm{~K}$ whose interplay with the microscopic magnetic behavior has been investigated by means of neutron diffraction, magnetization, pyroelectric, and relative dielectric permittivity measurements. We show that the onset of an E-type antiferromagnetic ordering of $\mathrm{Co}^{2+}$ and $\mathrm{Mn}^{4+}$ moments monitored by neutron diffraction provokes the noticeable jump of the relative dielectric permittivity $(\sim 9 \%)$ at about $40 \mathrm{~K}$. It is also shown that this jump can be totally suppressed by application of a magnetic field of $\mu_{0} \mathrm{H}=5 \mathrm{~T}$. Neutron experiments and magnetic measurements confirm that such a suppression leading to a significant magneto-dielectric effect is driven by a metamagnetic phase transition from the peculiar E-type ordering of $3 \mathrm{~d}$ moments into a collinear ferromagnetic order. Pyroelectric current measurements do not show any spontaneous electric polarization, so the large dielectric anomaly at zero field cannot be ascribed to a ferroelectric ordering. (C) 2015 AIP Publishing LLC.

[http://dx.doi.org/10.1063/1.4926403]
\end{abstract}

Materials with coupling between magnetic and electric order parameters are of great current interest coming from their potential technological impact in a wide field of applications and from the need for a deeper understanding of the mechanisms behind these couplings. ${ }^{1-3}$ Distorted perovskites such as manganites or nickelates containing small rare-earth atoms stabilize the formation of E-type magnetic structure in the transition-metal sublattice, i.e., up-up-down-down ( $\uparrow \downarrow \downarrow)$ spin chains along a specific crystallographic direction., Competitive magnetic interactions combining a small ferromagnetic nearest-neighbor exchange coupling and a large antiferromagnetic next-nearest-neighbor interaction is a critical ingredient for its occurrence. ${ }^{6}$ First principle calculations propose that compounds with E-type spin ordering are able to develop a finite ferroelectric polarization perpendicular to the $\uparrow \uparrow \downarrow \downarrow$ spin chains. The magnetic ordering induces cooperative shifts of oxygen atoms in a direction perpendicular to M-M path $(\mathrm{M}=\mathrm{Mn}$ or $\mathrm{Ni})$ in order to minimize the elastic energy. ${ }^{7,8}$ The close relationship between polarization and spin ordering makes these compounds members of the family of improper magnetic ferroelectrics. ${ }^{7}$ Recently, E-type structure has been found in a polycrystalline ceramic of $\mathrm{Lu}_{2} \mathrm{CoMnO}_{6}$, a double perovskite with a rock salt-like ordering of $\mathrm{Co}$ and $\mathrm{Mn}$ atoms. ${ }^{9}$ The magnetic order takes place along the crystallographic $c$-axis and it is coupled to the occurrence of an anomaly in the relative dielectric permittivity. In a single crystal of the same composition, ${ }^{10}$ the dielectric anomaly was observed along the $b$-axis, i.e., perpendicular to the spins chain direction in agreement with abovementioned

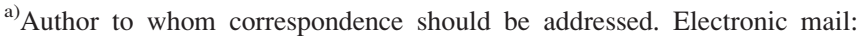
jbc@unizar.es
}

calculations for E-type structures. However, there is currently a strong controversy about the ferroelectric nature of this compound since the spontaneous electric polarization was observed in ceramic specimens ${ }^{9}$ but not in a single crystal. ${ }^{10}$ However, there is agreement in reporting a significant magnetodielectric effect in this material as the dielectric anomaly vanishes under application of an external magnetic field but no explanation has been given to this effect so far.

Recently, multiferroic properties have been also reported for two related compounds: $\mathrm{Y}_{2} \mathrm{CoMnO}_{6}$ and $\mathrm{Sm}_{2} \mathrm{CoMnO}_{6}{ }^{11,12}$ Both compounds exhibited a long range magnetic ordering and the ferroelectricity was inferred from pyroelectric measurements. An E-type magnetic ordering is suggested for both compounds without a detailed neutron diffraction study and this assignment is controversial. First of all, neutron diffraction refinements at low temperature revealed that $\mathrm{Y}_{2} \mathrm{CoMnO}_{6}$ does not show an E-type ordering, instead it shows a collinear ferromagnetic ordering similar to the La-based sample. . $^{13,14}$ Accordingly, the suggested E-type ordering in the Sm-based compound is also matter of discussion since double perovskites with smaller rare-earth cations, as $\mathrm{Y}^{3+}$ and $\mathrm{Tb}^{3+}$, also develop long range ferromagnetic ordering. ${ }^{13-15}$ Therefore, the origin of the reported pyroelectric properties in $\mathrm{Y}_{2} \mathrm{CoMnO}_{6}$ and $\mathrm{Sm}_{2} \mathrm{CoMnO}_{6}$ must be different to the behavior shown by $\mathrm{Lu}_{2} \mathrm{CoMnO}_{6}{ }^{9,10}$

In this letter, we report the synthesis and characterization of the $\mathrm{Yb}_{2} \mathrm{CoMnO}_{6}$, a double perovskite with a clear E-type magnetic ordering isostructural to $\mathrm{Lu}_{2} \mathrm{CoMnO}_{6}$. This material also shows an anomalous broad peak in the relative dielectric permittivity, coupled to the developing of long range E-type magnetic ordering, which can be suppressed by applying a magnetic field. Magnetic measurements and 
neutron powder diffraction experiments under various magnetic fields demonstrate that the dielectric anomaly is driven by the onset of the antiferromagnetic ordering and let us to discover a metamagnetic transition induced by the field that is responsible of the large magnetodielectric effect $(\sim 9 \%)$ of $\mathrm{Yb}_{2} \mathrm{CoMnO}_{6}$.

Polycrystalline samples of $\mathrm{Yb}_{2} \mathrm{CoMnO}_{6}$ can be synthesized by the conventional ceramic method. Stoichiometric amounts of $\mathrm{Yb}_{2} \mathrm{O}_{3}, \mathrm{Co}_{3} \mathrm{O}_{4}$, and $\mathrm{MnCO}_{3}$ were mixed, ground, and heated at $900{ }^{\circ} \mathrm{C}$ for $1 \mathrm{~d}$ with an intermediate grinding. The resulting powder was ground, pressed into pellets, and sintered at $1200^{\circ} \mathrm{C}$ in air for $2 \mathrm{~d}$ followed by a very slow cooling $\left(0.2^{\circ} \mathrm{C} / \mathrm{min}\right)$ down to $300^{\circ} \mathrm{C}$ before the samples are extracted from the furnace. This cooling improves both $\mathrm{Co} /$ Mn ordering and oxygen stoichiometry. ${ }^{16} \mathrm{X}$-ray diffraction patterns were collected at room temperature using a Rigaku $\mathrm{D} /$ max-B instrument with $\mathrm{Cu} \mathrm{K}_{\alpha}$ wavelength. Neutron diffraction experiments were carried out at the high-flux reactor of the ILL using the D1B $(\lambda=2.52 \AA)$ and D2B $(\lambda=1.59 \AA)$ instruments. All structural refinements were made using the Fullprof program. ${ }^{17}$

$\mathrm{X}$-ray diffraction at room temperature confirmed a single phase sample and showed that $\mathrm{Yb}_{2} \mathrm{CoMnO}_{6}$ adopts the monoclinic structure of a double perovskite $\left(P 2_{1} / n\right.$ space group) with a unit cell of $\mathrm{a}=5.1887(4) \AA, \mathrm{b}=5.5604(4) \AA$, $\mathrm{c}=7.4361(5) \AA$, and $\beta=90.33^{\circ}$ (1). High resolution neutron patterns (D2B) at $295 \mathrm{~K}$ also confirmed that our specimen exhibits an almost perfect ordering of $\mathrm{Co}$ and $\mathrm{Mn}$ atoms on the perovskite B-site as the refinement of occupancies yielded a composition of $94.2(8) \%$ of $\mathrm{Co}+5.8(8) \%$ of $\mathrm{Mn}$ at the Wyckoff $2 c$ site and the opposite ratio at the $2 d$ position. The average bond length in the $\mathrm{CoO}_{6}$ octahedron is 2.0603(9) $\AA$, very close to the value expected for $\mathrm{Co}^{2+}$ ion $(2.0985 \AA)$, while the average interatomic Mn-O distance is $1.9077(8) \AA$ also in agreement with the expected value for $\mathrm{Mn}^{4+}(1.9030 \AA)$.

The dc and ac magnetic properties of $\mathrm{Yb}_{2} \mathrm{CoMnO}_{6}$ were investigated from using a commercial Quantum Design SQUID magnetometer from 5 up to $295 \mathrm{~K}$. Magnetic hysteresis loops were obtained at selected temperatures between -5 and $5 \mathrm{~T}$. Figure 1(a) shows the temperature dependence of the dc-magnetization using zero-field-cooled (ZFC) and field-cooled (FC) conditions at an applied magnetic field of 100 Oe. The ZFC curve shows a peak at $31 \mathrm{~K}$ and a shoulder at $50 \mathrm{~K}$, whereas the FC branch shows the typical behavior of a ferromagnetic transition that may be ascribed to the spin canting induced by the external field. A strong divergence is observed between both curves and it begins at the shoulder temperature of the ZFC measurement. The magnetic transition temperature, estimated from the first turning point (minimum in the 1st derivative of $\mathrm{FC}$ curve), is around $51 \mathrm{~K}$. At higher temperatures, the magnetization obeys a Curie-Weiss law as shown in the inset of Fig. 1(a). The Weiss constant is positive, in agreement with the occurrence of ferromagnetic interactions. The calculated effective paramagnetic moment per unit formula is $8.94 \mu_{\mathrm{B}}$ in accordance to the expected contribution from $\mathrm{Yb}^{3+}\left(\mu_{\mathrm{th}}=4.54 \mu_{\mathrm{B}}\right)$, $\mathrm{Mn}^{4+}$ (spin only, $4.9 \mu_{\mathrm{B}}$ ), and $\mathrm{Co}^{2+}$ (experimental value of $\left.4.8 \mu_{\mathrm{B}}\right){ }^{18}$

The real component of the ac magnetic susceptibilitysee Figure 1(b)—shows a peak at $\sim 50 \mathrm{~K}$ whose intensity decreases as the frequency of the alternating field increases
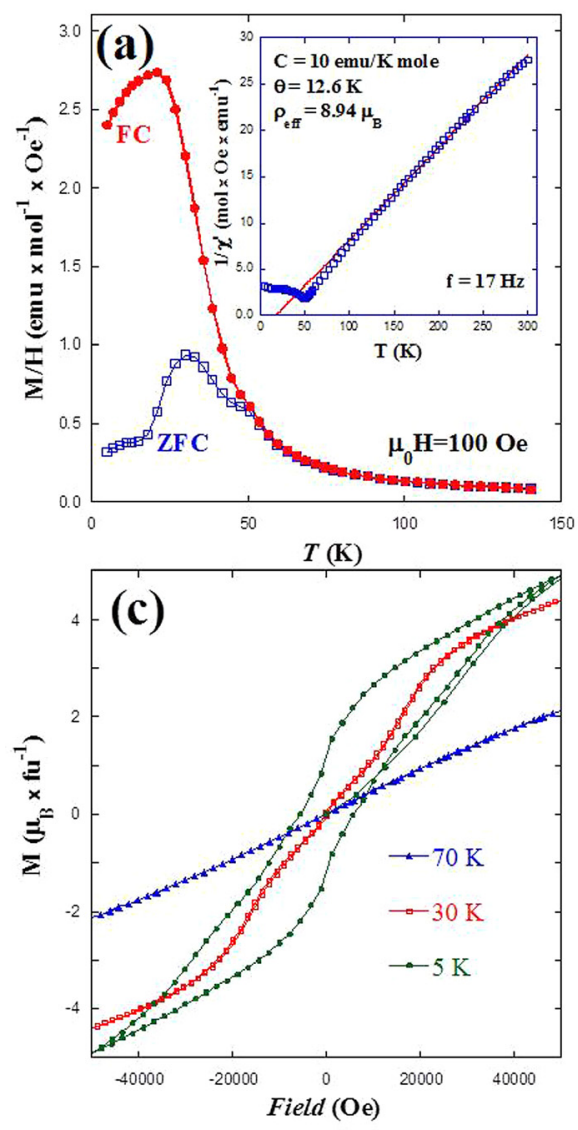
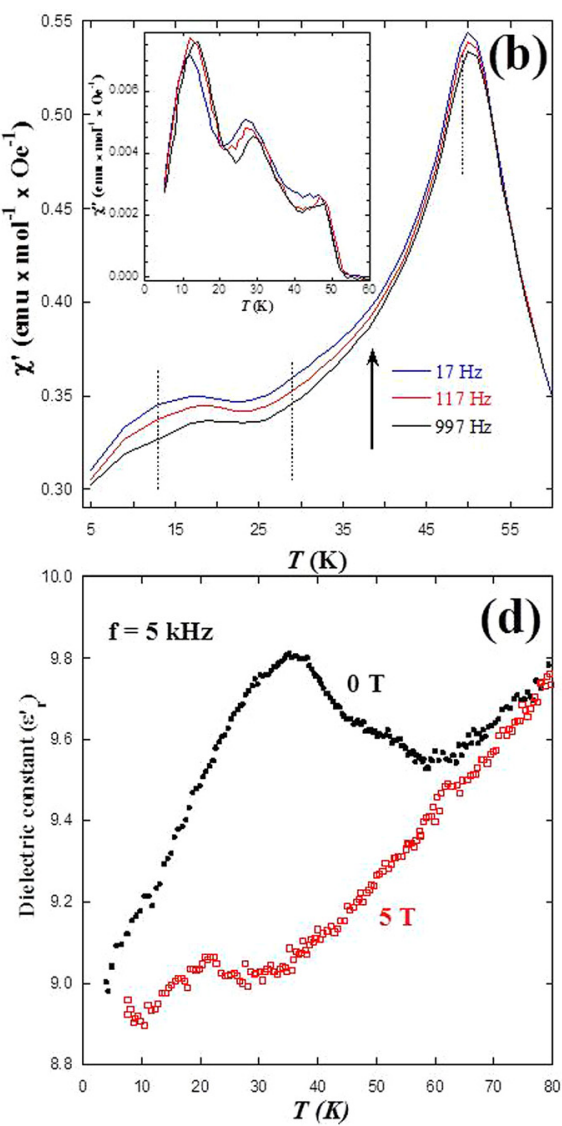

FIG. 1. (a) Temperature dependence of the dc magnetization for a field of $0.01 \mathrm{~T}$ in zero-field-cooled (ZFC) and field-cooled (FC) conditions. Inset: Inverse of the ac magnetic susceptibility $(\mathrm{f}=17 \mathrm{~Hz})$ vs temperature. Solid line is a linear fit (Curie-Weiss law). (b) Temperature dependence of the inphase component of ac magnetic susceptibility for a series of frequencies between 17 and $997 \mathrm{~Hz}$. Inset: Out-ofphase component. (c) Magnetization in $\mathrm{Yb}_{2} \mathrm{CoMnO}_{6}$ as a function of the external magnetic field for 5, 30, and $70 \mathrm{~K}$. (d) Temperature dependence of the real part of relative dielectric permittivity for $\mathrm{Yb}_{2} \mathrm{CoMnO}_{6}$ at $\mathrm{f}=5 \mathrm{kHz}$. 
but without significant shift of its position in the frequency range studied (17-997 Hz). At temperatures above the magnetic peak, no dynamic behavior is observed in accordance with a paramagnetic phase. However, the susceptibility becomes frequency dependent at low temperatures and a local broad maximum centered around $17.5 \mathrm{~K}$ is noticeable. The out-of-phase susceptibility-see inset of Fig. 1(b) shows three peaks at $\sim 13, \sim 28$, and $\sim 49 \mathrm{~K}$ indicating small changes (absorption) in the magnetic arrangement with decreasing temperature. The magnetic irreversibility in dc magnetization scans, in addition to the dynamic behavior in ac magnetic susceptibility, is usually ascribed to a glassy magnetic state such as spin- or cluster-glass. The occurrence of long range magnetic ordering revealed by neutron diffraction scattering (see below) rules out this only contribution.

The hysteresis curves of magnetization vs magnetic field reveal significant magnetization at low temperature as can be seen in Figure 1(c). These results indicate a ferromagneticlike behavior below $70 \mathrm{~K}$ under field. At $5 \mathrm{~K}$, the ferromagnetic transition induced by the field exhibits a wide hysteresis loop producing a notable remanent moment. However, saturation is not achieved up to $5 \mathrm{~T}$ in this loop. In fact, the magnetization at $5 \mathrm{~T}\left(4.9 \mu_{\mathrm{B}} / \mathrm{fu}\right)$ is below to the expected value of a fully magnetized lattice of $\mathrm{Mn}^{4+}$ and $\mathrm{Co}^{2+}\left(6 \mu_{\mathrm{B}}\right)$ $\mathrm{fu})$ and there is the potential contribution of the magnetic $\mathrm{Yb}^{3+}$. Both magnetization and coercive field increase with decreasing temperature. At $30 \mathrm{~K}$, the loop resembles that of a soft ferromagnet, while at $5 \mathrm{~K}$ is typical of a hard ferromagnetic material. The latter indicates either the occurrence of defects in the material able to pin the domain walls or an increase of the magnetic anisotropy with decreasing temperature. However, the most striking point in the loops is the presence of a turning point, clearly visible at $\sim 1.5 \mathrm{~T}$ at $30 \mathrm{~K}$, indicating a phase transition induced by the magnetic field. This feature is less noticeable in the loop at $5 \mathrm{~K}$ due to the large coercivity, but it may be responsible of the waspwaisted shape for the hysteresis cycle.

Relative dielectric permittivity $\left(\varepsilon_{\mathrm{r}}\right)$ of bulk $\mathrm{Yb}_{2} \mathrm{CoMnO}_{6}$ was measured at low temperature-see Figure 1(d) - on discshaped pellets of about $48 \mathrm{~mm}^{2}$ surface and $0.8 \mathrm{~mm}$ thick using silver paint as electrodes. These measurements were carried out as a function of temperature between 5 and $100 \mathrm{~K}$ with and without an external magnetic field of $5 \mathrm{~T}$ applied perpendicular to the ac electric field. The dielectric response was measured on heating using an impedance analyzer (Wayne Kerr Electronics 6500B) applying voltages with amplitude of $1 \mathrm{~V}$ and for frequencies between $100 \mathrm{~Hz}$ and $1 \mathrm{MHz}, \varepsilon_{\mathrm{r}}(\mathrm{T})$ shows an anomaly with the shape of rounded wide peak in the measurements without external magnetic field. No significant differences were found in function of the frequency. The anomaly begins at $\sim 59 \mathrm{~K}$, close to the onset of the magnetic transition-see Figure 1(a)—and it achieves a maximum at $\mathrm{T}=35 \mathrm{~K}$. The repetition of this measurement under an external field of $5 \mathrm{~T}$ leads to the anomaly fading and it results in a negative magnetocapacitance $(\sim 9 \%)$ for this material in this temperature range. These features agree with the previous reports in the related $\mathrm{Lu}_{2} \mathrm{CoMnO}_{6}$ compound suggesting a similar scenario for both samples. ${ }^{9}$ However, pyroelectric current measurements after cooling in an electric pole field of $20 \mathrm{kV} / \mathrm{cm}$ did not detect electric polarization in
$\mathrm{Yb}_{2} \mathrm{CoMnO}_{6}$ higher than $1 \mu \mathrm{C} / \mathrm{m}^{2}$ in agreement with the find-

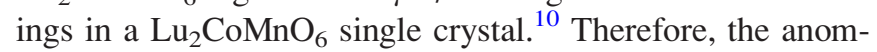
aly in the relative dielectric permittivity is not associated to the developing of significant ferroelectric ordering in $\mathrm{Yb}_{2} \mathrm{CoMnO}_{6}$.

In order to unravel the microscopic mechanism involved in the observed large magneto-dielectric response, we have performed neutron diffraction measurements under various magnetic fields. Figure 2(a) compares the neutron pattern at room temperature to the one collected at 25 and at $2 \mathrm{~K}$ at zero magnetic field. Magnetic contribution is subtle but clearly visible in the last two patterns. Long range magnetic ordering is developed in the Co-Mn sublattice below $50 \mathrm{~K}$. The magnetic peaks can be indexed using the propagation vector $\mathbf{k} \sim(0,0,1 / 2)$, and the refinements indicate that $\mathrm{Mn}$ and Co moments are mainly aligned along the $\mathrm{z}$-direction forming an E-type antiferromagnetic structure similar to the reported for $\mathrm{Lu}_{2} \mathrm{CoMnO}_{6}$. The propagation vector could be incommensurate but no definite conclusion can be obtained from powder neutron diffraction. First of all, magnetic peaks are rather broader than nuclear ones indicating a lower coherence length in the magnetic order. Second, some magnetic peaks have the typical asymmetrical shape of stacking faults, mainly the $\left(\begin{array}{lll}0 & 0 & 1\end{array}\right)$ peaks as can be seen in Figs. 2(b) and 2(c). These defects may be ascribed to antiphase boundaries in the E-type ordering along the $c$-axis. The large amount of defects in the magnetic coherence may account for the large coercivity observed in the hysteresis loops at low temperature.

The refinements are stabilized using the same magnetic moment for both $\mathrm{Co}^{2+}$ and $\mathrm{Mn}^{4+}$ suggesting a high spin configuration for both ions $(S=3 / 2)$, in agreement with the configuration observed in related double perovskites. ${ }^{15,19}$ However, refined magnetic moments are smaller than expected reaching a value of $1.68(7) \mu_{\mathrm{B}}$ /atom at $2 \mathrm{~K}$. Part of this difference can be ascribed to the abovementioned defects but it is quite likely that not the full Co-Mn sublattice is involved in the long range magnetic order. The magnetic ordering of $\mathrm{Yb}^{3+}$ moments occurs independently of the CoMn sublattice at lower temperatures. This $\mathrm{Yb}$ sublattice partially orders below $\sim 15 \mathrm{~K}$ and the magnetic peaks associated with this ordering are much broader than E-type peaks confirming partial disorder of $\mathrm{Yb}$ moments even at $2 \mathrm{~K}$ as can be seen in Fig. 2(d).

Due to its relevance, one of the most striking points in the magnetic behavior of $\mathrm{Yb}_{2} \mathrm{CoMnO}_{6}$ is the existence of a field induced transition at relatively low fields depending on temperature. Applying a magnetic field, several changes take place in the neutron diffraction patterns (see Fig. 3). First of all, the E-type magnetic peaks disappear while the intensity of some nuclear peaks rises as shown in Figs. 3(b) and 3(c). This increase is related to a long-range ferromagnetic ordering of the Co-Mn moments, so a transition from E-type to collinear ferromagnetic ordering is induced by an external field higher than $3 \mathrm{~T}$ at $2 \mathrm{~K}$. Second, the magnetic scattering associated to the $\mathrm{Yb}^{3+}$ order vanishes whilst the metamagnetic transition occurs indicating that the ferromagnetic coupling between $\mathrm{Co}^{2+}$ and $\mathrm{Mn}^{4+}$ moments prevents the longrange ordering of $\mathrm{Yb}^{3+}$ moments-see Fig. 3(d). 

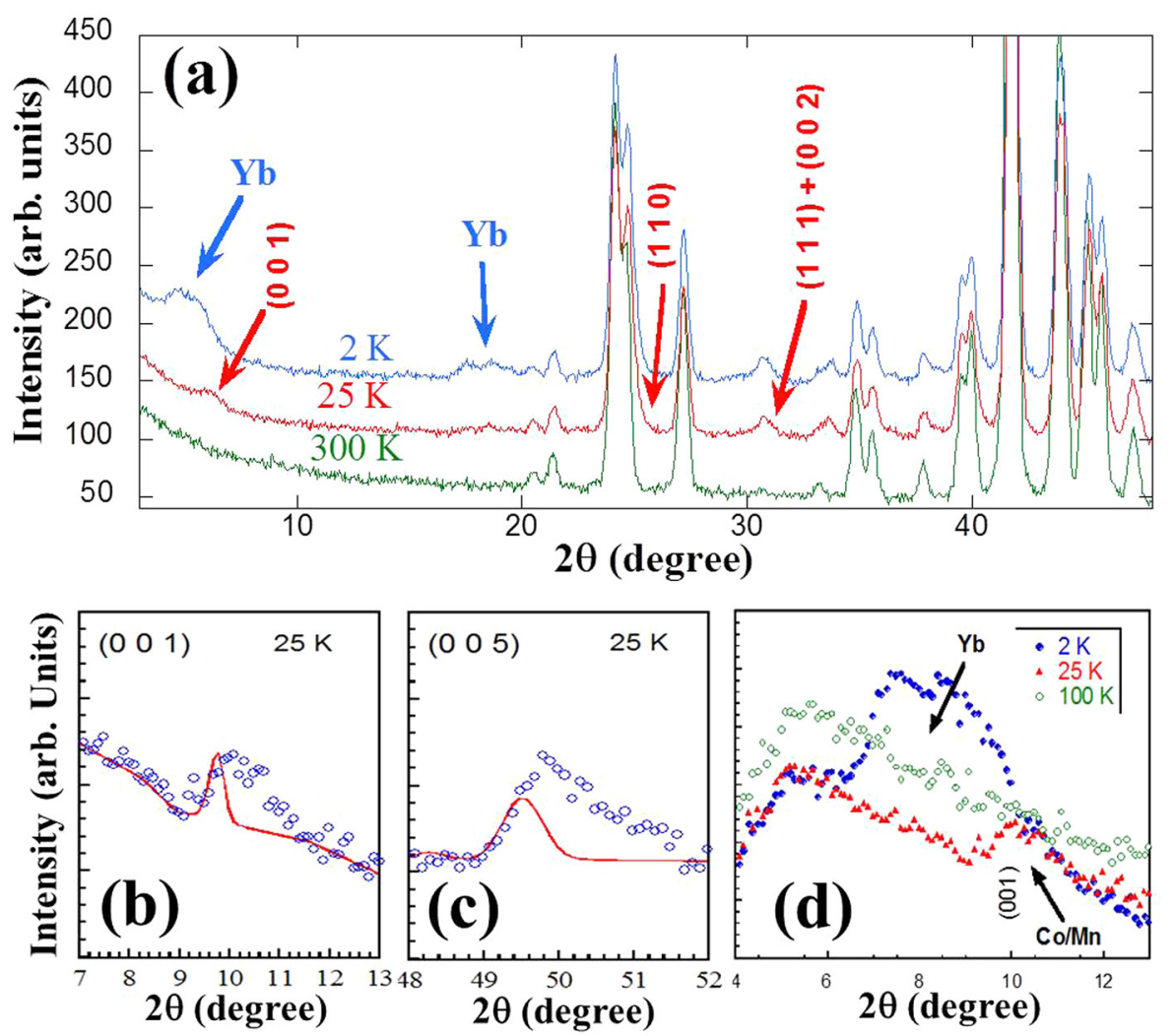

FIG. 2. (a) Neutron diffraction scans measured on D2B $(\lambda=1.59 \AA)$ for a series of representative temperatures: $100 \mathrm{~K}$ (paramagnetic region), $25 \mathrm{~K}$ (magnetic ordering of Co-Mn sublattice), and $2 \mathrm{~K}$ (emerging of $\mathrm{Yb}$ ordering). Arrows mark the main magnetic contribution with the magnetic reflections referred to the magnetic cell $(\mathrm{a} \times \mathrm{b} \times 2 \mathrm{c})$. (b) and (c) Comparison of the calculated reflections using natural width to the actual magnetic reflections $\left(\begin{array}{lll}0 & 0 & l\end{array}\right)$. (d) Comparison of representative neutron diffraction scans at low angles showing the emergency of magnetic contribution from $\mathrm{Yb}$ at $2 \mathrm{~K}$.

The refinement of the neutron patterns collected at $25 \mathrm{~K}$ and $5 \mathrm{~T}$ yields a ferromagnetic ordering in the $a c$-plane with a refined moment of 2.62(3) $\mu_{\mathrm{B}} /$ atom close to the expected value of a Co-Mn lattice fully saturated. Here, the refinement also agrees with the same magnetic moment for both atoms. This ferromagnetic ordering is usually found in some related double perovskites without external magnetic field. ${ }^{15,19}$ Finally, as the temperature increases the metamagnetic transition takes place at smaller magnetic fields - compare Fig. 3(b) with Fig. 3(c).

Summarizing, we have prepared and characterized a double perovskite, $\mathrm{Yb}_{2} \mathrm{CoMnO}_{6}$, isostructural to the reported
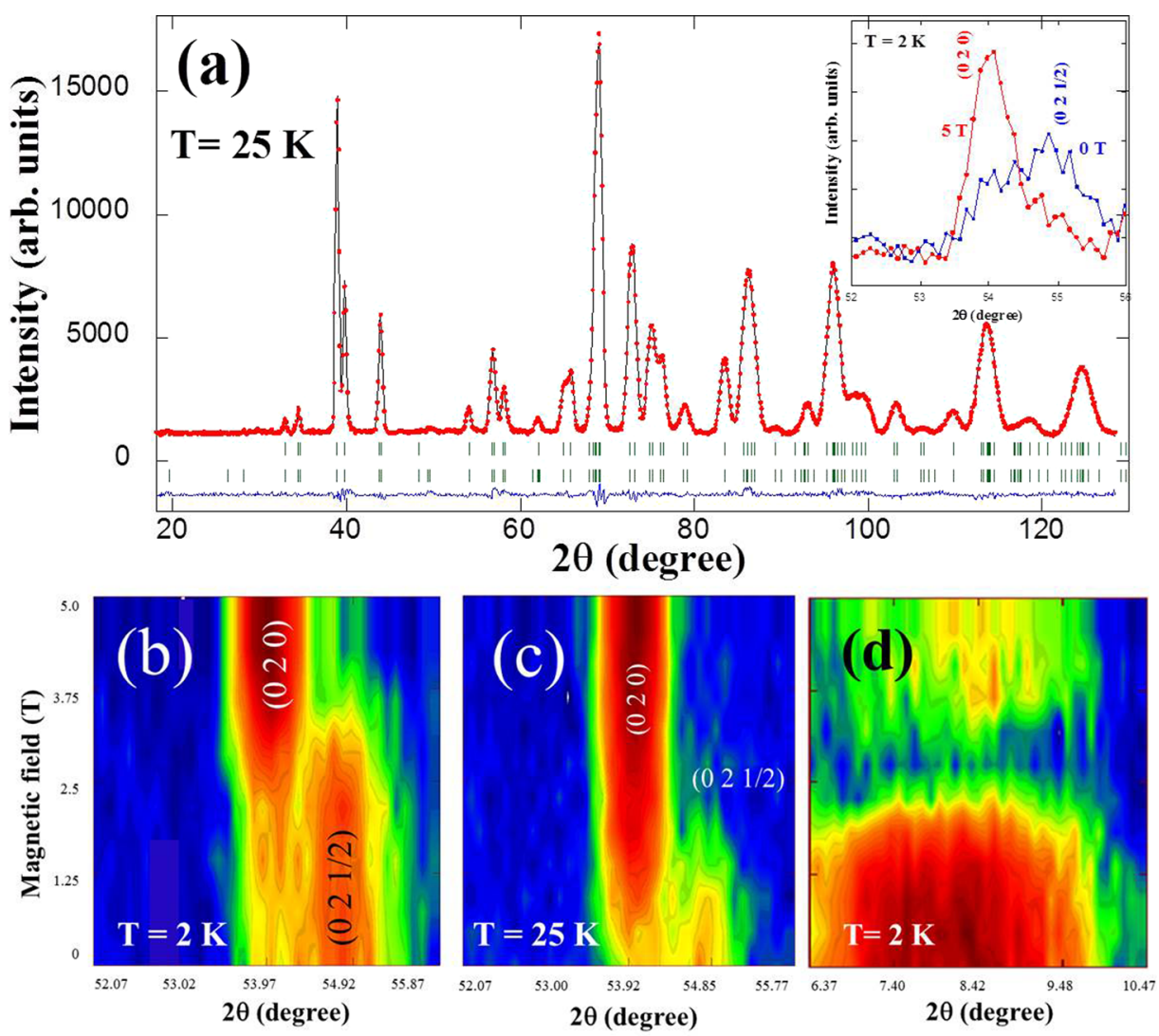

FIG. 3. (a) Refinement of the neutron powder diffraction pattern collected on D1B $(\lambda=2.52 \AA)$ for $\mathrm{Yb}_{2} \mathrm{CoMnO}_{6}$ at $25 \mathrm{~K}$ and $5 \mathrm{~T}$. Inset: Detail of the neutron patterns collected at 0 and $5 \mathrm{~T}$ and at $2 \mathrm{~K}$ evidencing changes in the magnetic scattering of two representative peaks. (b)-(d) Two dimensional plots representing neutron diffraction scans measured on D1B as a function of magnetic field in the region (as previous inset) of representative magnetic scattering from the Co-Mn sublattice at (b) $2 \mathrm{~K}$ and (c) $25 \mathrm{~K}$, and (d) region of representative magnetic scattering from $\mathrm{Yb}$ sublattice at $2 \mathrm{~K}$. The reflections are indexed in the frame of the crystallographic (nuclear) cell. 
multiferroic $\mathrm{Lu}_{2} \mathrm{CoMnO}_{6} . \mathrm{Yb}_{2} \mathrm{CoMnO}_{6}$ shows magnetic and electric transitions at low temperature identified by anomalies in the temperature dependence of magnetization and relative dielectric permittivity, respectively. The magnetic structure is antiferromagnetic of the E-type, which has been related to the developing of ferroelectricity in orthorhombic perovskites like $\mathrm{HoMnO}_{3} .{ }^{20}$ This compound shows a large negative magnetocapacitance and we demonstrated: (i) The emergence of a broad wide peak in the relative dielectric permittivity driven by the presence of E-type magnetic ordering. (ii) However, no ferroelectric polarization is observed in $\mathrm{Yb}_{2} \mathrm{CoMnO}_{6}$ by measuring the pyroelectric current suggesting that E-type order is not sufficient condition to develop ferroelectricity. In fact, the occurrence of pyroelectric effect in related compounds should be originated by a different mechanism. ${ }^{11,12}$ (iii) We have correlated its large magnetodielectric response with a metamagnetic transition. Indeed, the application of a moderate magnetic field substitutes the E-type order by a collinear ferromagnetic order which suppresses the excess of electric capacitance generated by the up-up-down-down spin chains.

We thank financial support from the Spanish MINECO (Project Nos. MAT2012-38213-C02-01 and MAT201238213-C02-02, cofunded by ERDF from EU) and Diputación General de Aragón (DGA-CAMRADS). One of us (J.A.R.-V.) acknowledges CSIC for a JAEdoc contract. We also acknowledge ILL and SPINS for beam time allocation and the use of Servicio General de Apoyo a la Investigación-SAI, Universidad de Zaragoza.
${ }^{1}$ J. F. Scott, Nat. Mater. 6, 256 (2007).

${ }^{2}$ A. P. Pyatakov and A. K. Zvezdin, Phys.-Usp. 55, 557 (2012).

${ }^{3}$ V. Castel and C. Brosseau, Appl. Phys. Lett. 92, 233110 (2008).

${ }^{4}$ A. Muñoz, M. T. Casáis, J. A. Alonso, M. J. Martínez-Lope, J. L. Martínez, and M. T. Fernández-Díaz, Inorg. Chem. 40, 1020 (2001).

${ }^{5}$ D. Okuyama, S. Ishiwata, Y. Takahashi, K. Yamauchi, S. Picozzi, K. Sugimoto, H. Sakai, M. Takata, R. Shimano, Y. Taguchi, T. Arima, and Y. Tokura, Phys. Rev. B 84, 054440 (2011).

${ }^{6}$ S.-W. Cheong and M. Mostovoy, Nat. Mater. 6, 13 (2007).

${ }^{7}$ I. A. Sergienko, C. Sen, and E. Dagotto, Phys. Rev. Lett. 97, 227204 (2006).

${ }^{8}$ S. Picozzi and C. Ederer, J. Phys.: Condens. Matter 21, 303201 (2009).

${ }^{9}$ S. Yañez-Vilar, E. D. Mun, V. S. Zapf, B. G. Ueland, J. S. Gardner, J. D. Thompson, J. Singleton, M. Sánchez-Andujar, J. Mira, N. Biskup, M. A. Señaris-Rodríguez, and C. D. Bautista, Phys. Rev. B 84, 134427 (2011).

${ }^{10}$ N. Lee, H. Y. Choi, Y. J. Jo, M. S. Seo, S. Y. Park, and Y. J. Choi, Appl. Phys. Lett. 104, 112907 (2014).

${ }^{11}$ G. Sharma, J. Saha, S. D. Kaushik, V. Siruguri, and S. Patnaik, Appl. Phys. Lett. 103, 012903 (2013).

${ }^{12}$ L. Wang, W. Zhou, D. Wang, Q. Cao, Q. Xu, and Y. Du, J. Appl. Phys. 117, 17D914 (2015).

${ }^{13}$ I. O. Troyanchuk, D. D. Khalyavin, J. W. Lynn, R. W. Erwin, Q. Huang, H. Szymczak, R. Szymczak, and M. Baran, J. Appl. Phys. 88, 360 (2000).

${ }^{14}$ H. S. Nair, R. Pradheesh, Y. Xiao, D. Cherian, S. Elizabeth, T. Hansen, T. Chatterji, and Th. Brückel, J. Appl. Phys. 116, 123907 (2014).

${ }^{15}$ J. Blasco, J. García, G. Subías, J. Stankiewicz, S. Lafuerza, J. A. Rodríguez-Velamazán, C. Ritter, and J. L. García-Muñoz, J. Phys.: Condens. Matter 26, 386001 (2014).

${ }^{16}$ T. Kyômen, R. Yamazaki, and M. Itoh, Chem. Mater. 15, 4798 (2003).

${ }^{17}$ J. Rodriguez-Carvajal, Physica B 192, 55 (1993).

${ }^{18}$ D. C. Jiles, Introduction to Magnetism and Magnetic Materials (Chapman \& Hall, London, 1996), p. 243.

${ }^{19}$ A. J. Barón-González, C. Frontera, J. L. García-Muñoz, B. Rivas-Murias, and J. Blasco, J. Phys.: Condens. Matter 23, 496003 (2011).

${ }^{20}$ N. Lee, Y. J. Choi, M. Ramazanoglu, W. Ratcliff II, V. Kiryukhin, and S.W. Cheong, Phys. Rev. B 84, 020101 (2011). 